\title{
HIGHER-ORDER KERNEL SEMIPARAMETRIC M-ESTIMATION OF LONG MEMORY
}

\author{
Peter M Robinson \\ Department of Economics, London School of Economics and Political Science \\ Marc Henry \\ Department of Economics, Columbia University, New York
}

Contents:

Abstract

1. Introduction

2. Higher-Order Kernel M-Estimation

3. Asymptotic Bias and Mean Squared Error

4. Partial Details of Derivations

5. A Special Case

6. Finite Sample Properties

7. Application to Intra-Day Foreign Exchange Volatility

References

Tables $1-5$

Discussion Paper

No. EM/02/436

September 2002
The Suntory Centre

Suntory and Toyota International Centres for

Economics and Related Disciplines

London School of Economics and Political Science Houghton Street

London, WC2A $2 \mathrm{AE}$

Tel.: 020-7955 6698

We thank two referees for helpful comments which have led to improvements in presentation. We also thank Fabrizio lacone for help in computing the theoretical bandwidths and theoretical mean squared errors in Tables $1-3$. The first author's research was supported by a Leverhulme Trust Personal Research Professorship and ESRC Grant R000238212. The second author's research was supported by an FRS Grant from IRES. 


\section{Abstract}

Econometric interest in the possibility of long memory has developed as a flexible alternative to, or compromise between, the usual short memory or unit root prescriptions, for example in the context of modelling cointegrating or other relationships and in describing the dependence structure of nonlinear functions of financial returns. Semiparametric methods of estimating the memory parameter can avoid bias incurred by misspecification of the short memory component. We introduce a broad class of such semiparametric estimates that also covers pooling across frequencies. A leading "Box-Club" sub-class, indexed by a single tuning parameter, interpolates between the popular local log periodogram and local Whittle estimates, leading to a smooth interpolation of asymptotic variances. The bias of these two estimates also differs to higher order, and we also show how bias, and asymptotic mean square error, can be reduced, across the class of estimates studied, by means of a suitable version of higher-order kernels. We thence calculate an optimal bandwidth (the number of low frequency periodogram ordinates employed) which minimizes this mean squared error. Finite sample performance is studied in a small Monte Carlo experiment, and an empirical application to intra-day foreign exchange returns is included.

Keywords: Long memory; semiparametric methods; higher-order kernel; $\mathrm{M}$ estimation; bias; mean squared error.

JEL No.: C22

(C) by the authors. All rights reserved. Short sections of text, not to exceed two paragraphs, may be quoted without explicit permission provided that full credit, including () notice, is given to the source.

Contact address: Professor Peter M Robinson, Department of Economics, London School of Economics and Political Science, Houghton Street, London WC2A 2AE. Email: p.m.robinson@lse.ac.uk 


\section{Introduction}

Semiparametric modelling has become popular in the investigation of possible long memory in economic and financial time series. A correct, fully parametric, model for autocorrelations over all lags, or equivalently for the spectral density over all frequencies, computed from a series of length $n$, advantageously produces $\sqrt{n}$ - consistent estimates of memory parameters. However, long memory is essentially a low frequency phenomenon, and semiparametric modelling consequently entails a parameterization of the spectral density only near frequency zero, or equivalently of autocorrelations at only long lags. Hence, there is potential to avoid the misspecification incurred by incorrect parametric modelling, such as by a fractionally integrated autoregressive moving average (FARIMA) model, when either the autoregressive or moving average order is under-specified, or both are over-specified; such misspecification can produce inconsistent estimates not only of the autoregressive and moving average parameters, which essentially describe short memory behaviour, but also of the memory parameter, which measures any long memory. Though semiparametric estimates are less-than- $\sqrt{n}-$ consistent, they do achieve consistency under broad conditions, without running such risk of misspecification, so their greater robustness might then be preferred, at least when $n$ is large.

A semiparametric approach is consistent with standard econometric practice nowadays, in connection with autocorrelation-consistent variance estimation and unit root time series analysis. Both involve $I(0)$ series, by which we mean covariance stationary (or asymptotically covariance stationary) series with spectral density that is finite and positive at frequency zero, and thus exhibit neither long memory nor antipersistence. In the first case, a statistic which can be approximated by a partial sum of functions of $I(0)$ series has asymptotic variance which involves a quantity that itself can be interpreted as the spectral density of a certain $I(0)$ series at frequency zero (see eg Hannan (1957)). In the second case, due to the preponderance of low frequency power in unit root series, correction for autocorrelation need entail estimation of the spectral density of the $I(0)$ input series at only zero frequency (see eg Phillips (1987)). In fact, criteria for semiparametric estimation of long memory have been used in testing the $I(0)$ hypothesis (see Lobato and Robinson (1998)), while autocorrelation-consistent variance estimates for the 
sample mean of a long memory series developed by Robinson (1994) involve semiparametric memory estimates.

We define the memory (or self-similarity) parameter $H$ of a covariance stationary time series $x_{t}, t=0, \pm 1, \ldots$, by means of the semiparametric representation

$$
f(\lambda) \sim C \lambda^{1-2 H}, \quad \text { as } \lambda \rightarrow 0+,
$$

for the spectral density $f(\lambda)$ of $x_{t}$, defined by $\operatorname{Cov}\left(x_{0}, x_{j}\right)=\int_{-\pi}^{\pi} f(\lambda) \cos j \lambda d \lambda$. In (1.1) " $\sim$ " indicates that the ratio of left- and right-hand sides tends to one, and

$$
\begin{aligned}
& 0<C<\infty \\
& 0<H<1 .
\end{aligned}
$$

When $0<H<0.5$ (so $f(0)=0$ ) there is said to be antipersistence or negative memory; when $H=0.5$ (so $0<f(0)<\infty$ ) there is said to be short memory; and when . $5<H<1$ (so $f(0)=\infty$ ) there is said to be long memory. As discussed above, (1.1) is silent about the behaviour of $f(\lambda)$ away from frequency zero; though it must be integrable, nonnegative, and even on $[-\pi, \pi]$, it need not be smooth and can have poles and/or zeroes. Indeed, Robinson (1995a,b) showed that two leading "semiparametric" estimates of $H$, based on an observed sequence $x_{t}, t=1, \ldots, n$, have desirable asymptotic properties in such a broad setting - these are the log periodogram estimate which originated in Geweke and Porter-Hudak (1983), and the semiparametric Gaussian or local Whittle estimate which originated in Künsch (1987).

Both estimates depend on a bandwidth parameter $m$, being the number of low frequency periodogram ordinates employed in the estimation, and both are $m^{1 / 2}$-consistent, where $m$ increases as $n$ increases, but more slowly. Indeed their mean squared errors are of order no smaller than $n^{-4 / 5}$ (Henry and Robinson (1996), Hurvich, Deo and Brodsky (1998)), compared to the $n^{-1}$ order of estimates based on a full parametric specification of $f(\lambda)$. This slow convergence is of concern, especially in macroeconomic series, where $n$ is unlikely to be very large. In particular, semiparametric memory estimates are useful in fractional cointegration analysis, where observables can be nonstationary, or even stationary, fractional processes, and cointegrating errors can be $I(0)$ or have long memory, for example in connection with purchasing power parity analysis and volume-volatility co-movements. Parametric 
modelling of dynamic structure is possible, but again there is danger of misspecification, and a semiparametric approach again fits in with ones used in standard cointegration analysis, which assumes that observables have unit roots and cointegrating errors are $I(0)$ (see e.g. Phillips (1991)). Note also that in cointegration or other analysis of nonstationary series, where first differences may have long memory or be antipersistent, nonstationary memory parameters arise. Here, in contrast with the superconsistent rates of, say, autoregressive coefficient estimates, semiparametric memory estimates are still only $\sqrt{m}$-consistent (see Velasco (2000)) (just as parametric estimates are still only $\sqrt{n}$-consistent). Thus a strong incentive for improving semiparametric memory estimates is identified.

Recently, Moulines and Soulier (1999), Hurvich and Brodsky (2001) have shown that a global version of log periodogram estimation, in which robustness to high frequency behaviour is achieved by a form of series expansion across all frequencies, rather than by omitting high frequencies, is capable of achieving a mean squared error of order $\log n / n$. However, this depends on $f(\lambda) / \lambda^{1-2 H}$ being globally analytic, across $[-\pi, \pi]$, which is true for FARIMA models but not, for example, for Gegenbauer models (see Gray, Zhang and Woodward, 1989), in which a spectral pole at some nonzero frequency is entertained, due to cyclic behaviour.

A striking feature of the asymptotic theory of Robinson (1995a,b) is that the regularity conditions correspond to those required in nonparametric spectral estimation for $I(0)$ series, due to the fact that $f(\lambda) \lambda^{2 H-1}$ is itself the spectrum of an $I(0)$ series. These conditions are purely local, unlike those of Moulines and Soulier (1999), Hurvich and Brodsky (2001). Now improvements to nonparametric spectral estimates are long established (see eg Parzen (1957), Cogburn and Davis (1974)), where in particular a lag window in a weighted sum of sample autocovariances is chosen to exploit a supposed degree of spectral smoothness (specifically the number of derivatives existing), in order to achieve a squared bias and a variance (and thence a meansquared error) of order $n^{-\kappa}$, where $\kappa<1$ but the index of smoothness $\kappa$ can approach arbitrarily close to 1 . In the frequency domain, this corresponds to periodogram averaging using a spectral window that behaves rather like the sort of higher-order kernel employed in nonparametric probability density estimation. Because a frequency-domain approach seems much more elegant than a time-domain one in our setting, we employ an analogous approach in 
estimating $H$, and are again able to achieve a mean-squared error of order $n^{-\kappa}, \kappa<1$, which can be nearly as good as that of Moulines and Soulier (1999), Hurvich and Brodsky (2001), but imposes only local assumptions. The kernels that arise in our case differ from those in the spectral density estimation problem, and while both are perforce negative over some part of their support, nothing corresponding to the worrying consequences of a negative spectral density emerges in our estimation of $H$.

One feature of the semiparametric long memory literature is the wide variety of estimates that has emerged. A number of these has the disadvantage of a nonstandard limit distribution for some or all $H$, or, if asymptotically normal for some values of $H$, having asymptotic variance depending on $H$ in a complicated way, while their convergence rate can also depend on $H$. This behaviour is perhaps associated with the relatively ad hoc character of such estimates, compared to the log periodogram and Gaussian semiparametric estimates, which both employ a suitable "whitening" (like classical least squares and maximum likelihood estimates) and are asymptotically normal for all $H$, with rates of convergence and (simple) asymptotic variances that are free of $H$. Nevertheless, the dichotomy in the literature between the log periodogram and semiparametric Gaussian estimate is still of some concern, as they tend to be treated individually, whereas they appear to be related and should be eligible for similar modification and, to some extent, be treatable simultaneously; the most obvious differences are that the former can be computed in closed form but is technically rather difficult to handle due to the logging of individual periodograms, whereas the second is only implicitly defined but more efficient. In the present paper we find that both are special cases of a class of M-estimates, so we can apply our higher-order kernel improvements to them both simultaneously (as well as to uncountably many other members of the class) thereby providing a unifying interpretation.

We focus on scalar stationary series satisfying (1.1), but it seems our higher-order kernel and M-estimation theory are extendable to multivariate series (cf Robinson (1995a), Lobato (1999)), to nonstationary series (cf Velasco (1999)), and to seasonal and cyclic long memory (cf Arteche (2000)), while the methods pursued by Moulines and Soulier (1999), Hurvich and Brodsky (2001) might also be included in a more general M-estimate class.

While we provide some justification, with relevant conditions, for our 
results, we do not attempt anything approaching full, rigorous proofs, or a full set of sufficient conditions. Reasonably complete proofs would be extremely lengthy. This is in part due to the implicit nature of many of our estimates, which require an initial consistency proof; in fact, as in other applications of higher-order order kernels, our estimate of $H$ solves first-order conditions but does not in general correspond to a global minimum of an objective function, so that only existence of a consistent root can be established. In general, dealing with actual means and variances of our estimates is very difficult. We adopt an approach of a familiar type, assuming consistency, approximating our estimates by relatively simple statistics by neglecting terms of smaller order in probability, and then approximating the moments of these simple statistics. We do not even provide full details of the latter step, because they are similar to ones in Robinson (1995a,b), Hurvich and Brodsky (1998), while a consistency proof would also follow in part that of Robinson (1995b); detailed proofs of this type thus no longer seem to have much novel interest in the semiparametric memory estimation context.

The following section introduces notation and our class of estimates. Aside from the $M$-estimation and higher-order kernel aspects discussed above, we consider two additional ones. One is the pooling across frequencies introduced by Robinson (1995a) to reduce the variance of log periodogram estimates; there is interest in the effect of such pooling on other members of our class. We also allow for different implementations of the estimates corresponding to alternative asymptotically equivalent versions of (1.1), a leading one being

$$
f(\lambda) \sim C\left(2 \sin \frac{\lambda}{2}\right)^{1-2 H}, \quad \text { as } \lambda \rightarrow 0+.
$$

For example, Geweke and Porter-Hudak's (1983) original version of the log periodogram estimate is based on (1.4), while Robinson's (1995a) is based on (1.1), but both have the same first-order asymptotic properties (see Robinson (1995a)). However, the errors in the approximations in (1.1) and (1.4) can differ from each other, and this can affect a higher-order analysis.

Section 3 presents results for bias, variance, mean squared error and optimal bandwidth. Their derivations are discussed in Section 4, and a special case is treated in Section 5. Monte Carlo simulations and an empirical application appear in, respectively, Sections 6 and 7. 


\section{Higher-Order Kernel M-Estimates}

For any integer $q \geq 1$ we introduce a user-chosen real-valued function $k_{q}(u)$, $0 \leq u \leq 1$, such that

$$
\int_{0}^{1} k_{q}(u) d u=1
$$

Also, defining

$$
U_{i q}=\int_{0}^{1}\{\log (u)+1\} u^{2 i} k_{q}(u) d u, \quad 0 \leq i \leq q,
$$

we suppose that $k_{q}(u)$ satisfies

$$
\begin{aligned}
& U_{i q}=0, \quad 0 \leq i \leq q-1, \\
& U_{q q} \neq 0 .
\end{aligned}
$$

We call $k_{q}(u)$ satisfying (2.1)-(2.4) a $q$ th order kernel. Perhaps the most analytically convenient class of $k_{q}(u)$ is

$$
k_{q}^{*}(u)=\sum_{j=0}^{q} a_{j} u^{2 j}, \quad 0 \leq u \leq 1,
$$

for which, in Section 5, we derive explicit formulae for the $a_{j}$ to satisfy (2.1)(2.4).

Denote by $\psi(z)$ a user-chosen, real-valued monotonic function. We shall stress in particular the "Box-Cox" class

$$
\begin{aligned}
& \psi_{\alpha}(z)=\frac{z^{\alpha}-1}{\alpha}, \quad z>0, \quad \alpha>0, \\
& \psi_{0}(z)=\log z, \quad z>0 .
\end{aligned}
$$

Introduce the periodogram

$$
I(\lambda)=\frac{1}{2 \pi n}\left|\sum_{t=1}^{n} x_{t} e^{i t \lambda}\right|^{2}
$$

based on a record of observations $x_{t}, 1 \leq t \leq n$, and for brevity denote

$$
I_{j}=I\left(\lambda_{j}\right), \quad \lambda_{j}=\frac{2 \pi j}{n},
$$


for integer $j$. To allow for pooling, for a fixed integer $J \geq 1$ introduce the $J$-averaged periodogram

$$
F_{j J}=\frac{1}{J} \sum_{l=j-J+1}^{j} I_{l}
$$

Denote by $m$ a user-chosen integer such that $1 \leq m<n / 2$. Our estimates use the $I_{j}$ for $1 \leq j \leq m$ only, or, more precisely, for $1 \leq j \leq[\mathrm{m} / J] J$, [.] denoting integer part.

Finally denote by $g(\lambda)$ a user-chosen non-negative function that is asymptotic to $\lambda$, to the extent that

$$
g(\lambda)=\lambda+G \lambda^{3}+o\left(\lambda^{3}\right), \quad \text { as } \lambda \rightarrow 0+.
$$

Two leading choices of $g(\lambda)$ are

$$
g^{(1)}(\lambda)=\lambda
$$

(cf. (1.1)), where $G=0$, and

$$
g^{(2)}(\lambda)=2 \sin \lambda / 2
$$

(cf. (1.4)), where $G=-\frac{1}{24}$.

We now define a $q$ th-order kernel $M$-estimate of $H, \widehat{H}_{q m}(\psi, g, k, J)$, as a solution of the equation

$$
\sum_{j}^{\prime} k_{q j} \nu_{q j}(g) \psi\left(F_{j J} g_{j}^{2 \widehat{H}_{q m}(\psi, g, k, J)-1}\right)=0,
$$

where $\sum_{j}^{\prime}$ throughout denotes a sum over $j=J, 2 J, \ldots,[\mathrm{m} / J] J$, and

$$
\begin{aligned}
& \nu_{q j}(g)=\log g_{j}-\frac{\sum_{j}^{\prime} k_{q j} \log g_{j}}{\sum_{j}^{\prime} k_{q j}}, \\
& g_{j}=g\left(\lambda_{j}\right), \quad k_{q j}=k_{q}\left(\frac{j}{m}\right) .
\end{aligned}
$$


The omission of frequency $\lambda_{0}=0$ (and $\lambda_{n}=2 \pi$ ) from (2.13) permits $x_{t}$ to have unknown mean, since $I_{j}$ is invariant to location shift for $1 \leq j<n / 2$.

To relate (2.13) to the existing literature on semiparametrically estimating $H$, we focus on the extreme choices of $\alpha$ in (2.6), (2.7), where $\alpha=0$ gives

$$
\widehat{H}_{q m}\left(\psi_{0}, g, k, J\right)=\frac{1}{2}\left\{1-\frac{\sum_{j}^{\prime} k_{q j} \nu_{q j}(g) \log F_{j J}}{\sum_{j}^{\prime} k_{q j} \nu_{q j}(g) \log g_{j}}\right\},
$$

and $\alpha=1$ gives

$$
\sum_{j}^{\prime} k_{q j} \nu_{q j}(g)\left\{F_{j J} g_{j}^{2 \widehat{H}_{q m}\left(\psi_{1}, g, k, J\right)-1}-1\right\}=0 .
$$

Using formulae in Section 5 below, (2.3) for $i=0, q=1$ implies $a_{1}=0$ in (2.5), so that, applying also (2.1),

$$
k_{1}^{*}(u)=a_{0}=1,
$$

to explain why we took $q \geq 1$ at the start of the section. We thus find that $\widehat{H}_{1 m}\left(\psi_{0}, g^{(2)}, k^{*}, 1\right)$ is the $\log$ periodogram estimate of Geweke and PorterHudak (1983), while $\widehat{H}_{1 m}\left(\psi_{0}, g^{(1)}, k^{*}, 1\right)$ is the log-periodogram estimate of Robinson (1995a), $\widehat{H}_{1 m}\left(\psi_{0}, g^{(1)}, k^{*}, J\right)$ is for $J>1$ the pooled log-periodogram estimate of Robinson (1995a), whereas $\widehat{H}_{1 m}\left(\psi_{1}, g^{(1)}, k^{*}, 1\right)$ is the local Whittle estimate of Künsch (1987), Robinson (1995b). (Strictly speaking, the latter estimate is defined as an extremum estimate, so if it falls on the boundary of the compact admissible parameter set it need not solve the first-order conditions (2.17).) Choosing $\alpha$ between 0 and 1 interpolates between these estimates, and turns out to produce an intermediate asymptotic variance (when $J=1$, between $\pi^{2} / 24$ for $\alpha=0$ and $1 / 4$ for $\alpha=1$ ). On the other hand, for given $\psi$, a higher-order kernel, in particular taking $q \geq 2$ in (2.5), has the potential to reduce asymptotic bias, and thence asymptotic meansquared error. The choice of $g$, among those satisfying (2.10), does not affect asymptotic variance but it can affect bias and thus mean squared error, even possibly in terms of rate of convergence. 


\section{Asymptotic Bias and Mean Squared Error}

We refine (1.1) by assuming that

$$
f(\lambda)=C g^{1-2 H}(\lambda) h(\lambda),
$$

where (1.2), (1.3) and (2.10) hold, along with

$$
h(\lambda)-1-\sum_{i=1}^{q} \frac{h_{i} \lambda^{2 i}}{(2 i) !}=O\left(\lambda^{2(q+1)}\right), \quad \text { as } \lambda \rightarrow 0+.
$$

Note that $h_{i}$ is the $2 i$ th derivative of $h(\lambda)$ at $\lambda=0$, so that (3.2) is tantamount to assuming that $f(\lambda) / g^{1-2 H}(\lambda)$ is $2 q$-times continuously differentiable at $\lambda=0$. In the central limit theory of Robinson $(1995 \mathrm{a}, \mathrm{b})$ for standard log periodogram and local Whittle estimates, $q$ was allowed to be a continuous smoothness parameter. However for pure FARIMA processes $q$ can be arbitrarily large in (3.2), and whenever $q \geq 2$ we can exploit (3.2) by the $q$ th order kernel $k_{q}(u)$ to reduce the order of magnitude of the bias, and thence that of the mean squared error.

For a given $f(\lambda)$, changing the choice of $g(\lambda)$ in (3.1) will affect the $h_{i}$, in a manner that depends also on $H$. For example suppose we commence from the form

$$
f(\lambda)=C g^{(2)}(\lambda)^{1-2 H} h^{(2)}(\lambda)
$$

where $h^{(2)}(\lambda)$ is the spectrum of an autoregressive moving average (ARMA) process, so that $x_{t}$ is a FARIMA. Then we can instead write

$$
f(\lambda)=C g^{(1)}(\lambda)^{1-2 H} h^{(1)}(\lambda),
$$

where $h^{(1)}(\lambda)=\left\{g^{(2)}(\lambda) / g^{(1)}(\lambda)\right\}^{1-2 H} h^{(2)}(\lambda)$. Indeed in case of a $\operatorname{FARIMA}(0$, $H-1 / 2,0)$ process, we have $h^{(2)}(\lambda) \equiv 1$, all of whose derivatives are zero, whereas $h^{(1)}(\lambda)$ has nonzero derivatives. For simplicity we will later often suppress reference to $g$ in our notation.

As acknowledged in Section 1, we do not list full regularity conditions but it is helpful to indicate what may constitute a large part of a set of sufficient conditions. We take $x_{t}$ to be Gaussian. We take $m$ to satisfy at least

$$
m \rightarrow \infty, \quad m=O\left(n^{\frac{4 q}{4 q+1}}\right), \text { as } n \rightarrow \infty .
$$


For simplicity no trimming (omitting of contributions from the very lowest Fourier frequencies) was allowed for in (2.13). We can trim, and it may facilitate proofs for our general class, noting that Robinson (1995a) required trimming in his central limit theorem for $\widehat{H}_{1 m}\left(\psi_{0}, g^{(1)}, k^{*}, J\right)$. However, under somewhat different conditions, for the alternative log-periodogram estimate $\widehat{H}_{1 m}\left(\psi_{0}, g^{(2)}, k^{*}, 1\right)$, Hurvich, Deo and Brodsky (1998) avoided trimming, while no trimming was required by Robinson (1995b) for the local Whittle estimate $\widehat{H}_{1 m}\left(\psi_{1}, g^{(1)}, k^{*}, 1\right)$. Some smoothness in $\psi(z)$ is necessary in order to demonstrate improvements as $q$ increases, and for convenience we take it to be infinitely differentiable, such that, with $\psi^{(u)}(z)$ denoting $u$ th derivative,

$$
E\left|\left(C Z_{J}\right)^{u} \psi^{(u)}\left(C Z_{J}\right)\right| \leq K<\infty, \quad u \geq 1,
$$

where $K$ is a positive constant independent of $u$, and $Z_{J}$ denotes a $\chi_{2 J}^{2} /(2 J)$ random variable. Notice that in case of (2.6), (2.7), the inequalities (3.5) are automatically satisfied - see (5.7) below. We take $k_{q}(u)$ to be boundedly differentiable except perhaps at finitely many points. Define, for $q \geq 1$,

$$
V_{q}=\int_{0}^{1}\{\log (u)+1\}^{2} k_{q}(u) d u, \quad W_{q}=\int_{0}^{1}\{\log (u)+1\}^{2} k_{q}(u)^{2} d u,
$$

and for $1 \leq i \leq q$,

$$
\begin{aligned}
Y_{i q} & =\int_{0}^{1} u^{2+2 i} k_{q}(u) d u-\int_{0}^{1} u^{2} k_{q}(u) d u \int_{0}^{1} u^{2 i} k_{q}(u) d u \\
P_{i J}(\psi) & =(2 \pi)^{2 i} \sum_{u=1}^{i} \frac{1}{u !} E\left(\left(C Z_{J}\right)^{u} \psi^{(u)}\left(C Z_{J}\right)\right) \sum_{r_{1}+\ldots+r_{u}=i}\left(\prod_{l=1}^{u} \frac{h_{r_{l}}}{\left(2 r_{l}\right) !}\right), \\
Q_{J}(\psi) & =J V\left(\psi\left(C Z_{J}\right)\right), \\
R_{J}(\psi) & =2 E\left(C Z_{J} \psi^{(1)}\left(C Z_{J}\right)\right) .
\end{aligned}
$$

Bearing in mind that in general $\widehat{H}_{q m}(\psi, g, k, J)$ is only implicitly-defined, and remarks in Section 1, we consider asymptotic bias and asymptotic variance based on a formal expansion, first approximating $\widehat{H}_{q m}(\psi, g, k, J)-H$ by $-A / B$, where

$$
\begin{aligned}
A & =\frac{J}{m} \sum_{j}^{\prime} k_{q j} \nu_{q j}(g) \psi\left(F_{j J} g_{j}^{2 H-1}\right), \\
B & =\frac{2 J}{m} \sum_{j}^{\prime} k_{q j} \nu_{q j}(g) \log \left(g_{j}\right) F_{j J} g_{j}^{2 H-1} \psi^{(1)}\left(F_{j J} g_{j}^{2 H-1}\right) .
\end{aligned}
$$


Following arguments used in the discussion of $A$ in the following section, we have

$$
B=R_{J}(\psi) V_{q}+o_{p}(1) .
$$

We then take

$$
\begin{aligned}
A B & =\text { Asybias }\left\{\widehat{H}_{q m}(\psi, g, k, J)\right\}=-\frac{\operatorname{Asymea}\{A\}}{R_{J}(\psi) V_{q}} \\
A V & =\operatorname{Asyvar}\left\{\widehat{H}_{q m}(\psi, g, k, J)\right\}=\frac{\operatorname{Asyvar}\{A\}}{R_{J}^{2}(\psi) V_{q}^{2}} \\
A M & =\text { Asymse }\left\{\widehat{H}_{q m}(\psi, g, k, J)\right\}=\frac{\operatorname{Asymse}\{A\}}{R_{J}^{2}(\psi) V_{q}^{2}}
\end{aligned}
$$

where Asymea $\{A\}$ and Asyvar $\{A\}$ represent the leading terms in the mean and variance of an approximation to $A$ that neglects terms of small order in probability, and Asymse $\{A\}=\operatorname{Asyvar}\{A\}+(\text { Asymea }\{A\})^{2}$. We have

$$
\begin{aligned}
A B= & -\frac{U_{q q} P_{q J}(\psi)(m / n)^{2 q}+(2 \pi)^{2} G Y_{1 q} P_{1 J}(\psi)(m / n)^{4}}{R_{J}(\psi) V_{q}}, \\
A V= & \frac{Q_{J}(\psi) W_{q}}{\left\{R_{J}(\psi) V_{q}\right\}^{2}} \frac{1}{m} \\
A M= & {\left[Q_{J}(\psi) W_{q} / m+\left\{U_{q q} P_{q J}(\psi)\left(\frac{m}{n}\right)^{2 q}\right.\right.} \\
& \left.\left.+(2 \pi)^{2} G Y_{1 q} P_{1 J}(\psi)\left(\frac{m}{n}\right)^{4}\right\}^{2}\right] /\left\{R_{J}(\psi) V_{q}\right\}^{2} .
\end{aligned}
$$

Then $A M$ is minimized by $\widehat{m}=m_{q}(\psi, q, k, J)$, given by

$$
\begin{aligned}
\widehat{m} & =\left[\frac{Q_{J}(\psi) W_{q}}{4 q U_{q q}^{2} P_{q J}^{2}(\psi)}\right]^{1 /(4 q+1)} n^{4 q /(4 q+1)}, G=0 \text { or } q=1, \\
& =\frac{\left\{Q_{J}(\psi) W_{q} / 8\right\}^{1 / 9}}{\left\{U_{22} P_{2 J}(\psi)+(2 \pi)^{2} G P_{1 J}(\psi) Y_{12}\right\}^{2 / 9}} n^{8 / 9}, G \neq 0, q=2 \\
& =\left[\frac{Q_{J}(\psi) W_{q}}{32 \pi^{2} G^{2} P_{1 J}^{2}(\psi) Y_{1 q}^{2}}\right]^{1 / 9} n^{8 / 9}, \quad G \neq 0 \text { and } q>2
\end{aligned}
$$


The minimized MSE is given by

$$
\begin{aligned}
& \frac{(4 q+1)\left[\left\{Q_{J}(\psi) W_{q}\right\}^{4 q} U_{q q}^{2} P_{q J}^{2}(\psi)\right]^{1 /(4 q+1)}}{(4 q)^{4 q /(4 q+1)}\left\{R_{J}(\psi) V_{q}\right\}^{2} n^{4 q /(4 q+1)}}, G=0 \text { or } q=1(3.15) \\
& \frac{9\left\{Q_{J}(\psi) W_{q}\right\}^{8 / 9}\left\{U_{22} P_{2 J}(\psi)+(2 \pi)^{2} G P_{1 J}(\psi) Y_{12}\right\}^{2 / 9}}{8^{8 / 9}\left\{R_{J}(\psi) V_{2}\right\}^{2} n^{8 / 9}} \\
G \neq & 0 \text { and } q=2 \\
& \frac{9\left[\left\{Q_{J}(\psi) W_{q}\right\}^{8}\left\{(2 \pi)^{2} G P_{1 J}(\psi) Y_{1 q}\right\}^{2}\right]^{1 / 9}}{8^{8 / 9}\left\{R_{J}(\psi) V_{q}\right\}^{2} n^{8 / 9}} \\
G \neq & 0 \text { and } q>2 .
\end{aligned}
$$

The cases described in (3.13), (3.14), (3.16), (3.17) are purely due to $G \neq 0$ in (2.10), that is, not taking $g(\lambda)=\lambda$. On the other hand, if we choose some other $g(\lambda)$, such as $2 \sin (\lambda / 2)(2.12)$, the problem is avoided by replacing the factor $u^{2 i}$ by $g\left(u^{2 i}\right)$ in our specification of the $U_{i q}$ in (2.2) (thereby, with (2.3), altering the precise specification of the $k_{q}(u)$ ) and considering an expansion for $h(\lambda)$ in powers of $g(\lambda)$ rather than $\lambda$ in (3.2). We have stressed the specification (2.2) because of the consequent ability to determine $k_{q}(u)$ analytically, specifically the $a_{j}$ in (2.5), and on the other hand we feel the implications of not thereby 'matching' $g(\lambda)$ with $k_{q}(u)$ are worth noting.

We mention connections with other work. Graf (1983) discussed $M$ estimation of parametric long memory models in the frequency domain, employing information from the entire Nyqvist band, and with a heuristic treatment of asymptotic theory. On the other hand, Andrews and Guggenberger (2000) reduce the bias, and hence the mean squared error, of the version of the log periodogram estimate of Robinson (1995a). They do not employ higher order kernels, but instead incorporate suitable additional variables in the log periodogram regression, to derive results that correspond to ours, providing rigorous proofs in their more specialized setting. A similar achievement is due to Andrews and Sun (2001), who propose a local polynomial modification to local Whittle estimation. This disadvantageously increases the dimension of the nonlinear optimization problem, though the additional parameter estimates are useful in optimal choice of $m$. 


\section{Partial Details of Derivations}

We focus on the (semi-heuristic) derivation of Asymea $\{A\}$ and Asyvar $\{A\}$, and thence Asymse $\{A\}$, in (3.6)-(3.8). Write $A=A_{1}+A_{2}+A_{3}+A_{4}+A_{5}$, where

$$
\begin{aligned}
A_{1}= & \frac{J}{m} \sum_{j}^{\prime} b_{q j}\left\{E \psi\left(Z_{J} f_{j} g_{j}^{2 H-1}\right)-E \psi\left(C Z_{J}\right)\right\}, \\
A_{2}= & \frac{J}{m} \sum_{j}^{\prime} b_{q j}\left\{\psi\left(2 \pi C F_{\varepsilon j J}\right)-E \psi\left(2 \pi C F_{\varepsilon j J}\right)\right\}, \\
A_{3}= & \frac{J}{m} \sum_{j}^{\prime} b_{q j}\left\{\psi\left(2 \pi F_{\varepsilon j J} f_{j} g_{j}^{2 H-1}\right)-\psi\left(2 \pi C F_{\varepsilon j J}\right)\right. \\
& \left.-E \psi\left(Z_{J} f_{j} g_{j}^{2 H-1}\right)+E \psi\left(C Z_{J}\right)\right\}, \\
A_{4}= & \frac{J}{m} \sum_{j}^{\prime} b_{q j}\left\{\psi\left(F_{j J} g_{j}^{2 H-1}\right)-\psi\left(\frac{2 \pi}{J} \sum_{l=j-J+1}^{j} I_{\varepsilon l} f_{l} g_{j}^{2 H-1}\right)\right\}, \\
A_{5}= & \frac{J}{m} \sum_{j}^{\prime} b_{q j}\left\{\psi\left(\frac{2 \pi}{J} \sum_{l=j-J+1}^{j} I_{\varepsilon l} f_{l} g_{j}^{2 H-1}\right)-\psi\left(2 \pi F_{\varepsilon j J} f_{j} g_{j}^{2 H-1}\right)\right\},
\end{aligned}
$$

where we write $f_{j}=f\left(\lambda_{j}\right), b_{q j}=k_{q j} \nu_{q j}, \nu_{q j}=\nu_{q j}(g)$, define

$$
I_{\varepsilon j}=\frac{1}{2 \pi n}\left|\sum_{t=1}^{n} \varepsilon_{t} e^{i t \lambda_{j}}\right|^{2}, F_{\varepsilon j J}=\frac{1}{J} \sum_{l=j-J+1}^{j} I_{\varepsilon l},
$$

where the $\varepsilon_{t}$ are $N I D(0,1)$, and note that $E \psi\left(2 \pi C F_{\varepsilon j J}\right)=E \psi\left(C Z_{J}\right)$ and, from (2.17), $\sum_{j}^{\prime} k_{q j} \nu_{q j}=0$.

We later argue that $A_{4}$ and $A_{5}$ can be neglected, in the sense of being $o_{p}\left(m^{-1 / 2}\right)$, so we consider $A_{1}+A_{2}+A_{3}$. By elementary inequalities,

$$
\begin{aligned}
E\left(A_{1}+A_{2}+A_{3}\right)^{2} & \left.=E\left(A_{1}+A_{2}\right)^{2}+2 E\left\{\left(A_{1}+A_{2}\right) A_{3}\right)\right\}+E\left(A_{3}^{2}\right) \\
& =A_{1}^{2}+V\left(A_{2}\right)+O\left(\left\{A_{1}^{2}+V\left(A_{2}\right)\right\}^{1 / 2}\left\{V\left(A_{3}\right)\right\}^{1 / 2}+V\left(A_{3}\right)\right),
\end{aligned}
$$

because $A_{1}$ is nonstochastic and $A_{2}$ and $A_{3}$ have zero means.

Consider first $A_{1}$. In the formal expansion

$$
\psi(a b)=\psi(a)+\sum_{l=1}^{\infty} a^{l} \psi^{(l)}(a) \frac{(b-1)^{l}}{l !},
$$


we have, taking $a=C Z_{J}, b=h\left(\lambda_{j}\right)$,

$$
\begin{aligned}
A_{1}= & \frac{J}{m} \sum_{j}^{\prime} b_{q j} \sum_{l=1}^{\infty} E\left(\left(C Z_{J}\right)^{l} \psi^{(l)}\left(C Z_{J}\right)\right) \frac{\left(h\left(\lambda_{j}\right)-1\right)^{l}}{l !} \\
= & \frac{J}{m} \sum_{j}^{\prime} b_{q j} \sum_{l=1}^{\infty} E\left(\left(C Z_{J}\right)^{l} \psi^{(l)}\left(C Z_{J}\right)\right) \frac{1}{l !}\left(\sum_{i=1}^{q} \frac{h_{i} \lambda_{j}^{2 i}}{(2 i) !}\right)^{l}+o\left(\left(\frac{m}{n}\right)^{2 q}\right) \\
= & \frac{J}{m} \sum_{j}^{\prime} b_{q j} \sum_{i=1}^{q} \lambda_{j}^{2 i} \sum_{u=1}^{i} \frac{1}{u !} E\left(\left(C Z_{J}\right)^{u} \psi^{(u)}\left(C Z_{J}\right)\right) \\
& \times \sum_{r_{1}+\ldots+r_{u}=i}\left(\prod_{l=1}^{u} \frac{h_{r_{l}}}{\left(2 r_{l}\right) !}\right)+o\left(\left(\frac{m}{n}\right)^{2 q}\right) .
\end{aligned}
$$

Now for $i=1, \ldots, q$,

$$
\frac{J}{m} \sum_{j}^{\prime} b_{q j}\left(\frac{j}{m}\right)^{2 i}=U_{i q}+a_{1 i}+a_{2 i},
$$

where

$$
\begin{aligned}
& a_{1 i}=\frac{J}{m} \sum_{j}^{\prime} k_{q j}\left\{\log \left\{g_{j} / \lambda_{j}\right\}-\frac{\sum_{j}^{\prime} k_{q j} \log \left\{g_{j} / \lambda_{j}\right\}}{\sum_{j}^{\prime} k_{q j}}\right\}\left(\frac{j}{m}\right)^{2 i}, \\
& a_{2 i}=\frac{J}{m} \sum_{j}^{\prime} k_{q j}\left\{\log \left(\frac{j}{m}\right)-\frac{\sum_{j}^{\prime} k_{q j} \log (j / m)}{\sum_{j}^{\prime} k_{q j}}\right\}\left(\frac{j}{m}\right)^{2 i}-U_{i q} .
\end{aligned}
$$

In case $g(\lambda)$ is given by (2.11), so $G=0$ in (2.10), we have $a_{1 i} \equiv 0$. Otherwise, including under (2.12),

$$
a_{1 i}=\lambda_{m}^{2} G Y_{i q}+o\left(\left(\frac{m}{n}\right)^{2}\right) .
$$

On the other hand, proceeding as in the proof of Lemma 2 of Robinson (1995b),

$$
a_{2 i}=O\left(\frac{\log m}{m}\right) .
$$


Thus (4.1) is

$$
\begin{aligned}
& \sum_{i=1}^{q}\left\{U_{i q}+G Y_{i q} \lambda_{m}^{2}+o\left(\left(\frac{m}{n}\right)^{2}\right)+O\left(\frac{\log m}{m}\right)\right\} \lambda_{m}^{2 i} \\
& \times \sum_{u=1}^{i} \frac{1}{u !} E\left(\left(C Z_{J}\right)^{u} \psi^{(u)}\left(C Z_{J}\right)\right) \sum_{r_{1}+\ldots+r_{u}=i}\left\{\prod_{l=1}^{u} \frac{h_{r_{l}}}{\left(2 r_{l}\right) !}\right\}+o\left(\left(\frac{m}{n}\right)^{2 q}\right) \\
&=U_{q q} P_{q J}(\psi)\left(\frac{m}{n}\right)^{2 q}+(2 \pi)^{2} G Y_{1 q} P_{1 J}(\psi)\left(\frac{m}{n}\right)^{4}+o\left(\left(\frac{m}{n}\right)^{2 q}+\left(\frac{m}{n}\right)^{4}\right)+O\left(\frac{m \log m}{n^{2}}\right) .
\end{aligned}
$$

It follows that

$$
\begin{aligned}
A_{1} & =U_{q q} P_{q J}(\psi)\left(\frac{m}{n}\right)^{2 q}+o\left(\left(\frac{m}{n}\right)^{2 q}\right)+O\left(\frac{1}{n}\right), \quad G=0 \text { or } q=1, \\
& =\left\{U_{22} P_{2 J}(\psi)+(2 \pi)^{2} G Y_{12} P_{1 J}(\psi)\right\}\left(\frac{m}{n}\right)^{4}+o\left(\left(\frac{m}{n}\right)^{4}\right)+O\left(\frac{1}{n}\right), \quad G \neq 0, q=2, \\
& =(2 \pi)^{2} G Y_{1 q} P_{1 J}(\psi)\left(\frac{m}{n}\right)^{4}+o\left(\left(\frac{m}{n}\right)^{4}\right)+O\left(\frac{1}{n}\right), \quad G \neq 0, q>2 .
\end{aligned}
$$

Next, because the $2 \pi F_{\varepsilon j J}$ are iid $\chi_{2 J}^{2} /(2 J)$ variates,

$$
\begin{aligned}
V\left(A_{2}\right) & =\frac{1}{m} Q_{J}(\psi) \frac{J}{m} \sum_{j}^{\prime} b_{q j}^{2} \\
& =\frac{1}{m} Q_{J}(\psi) W_{q}+o\left(\frac{1}{m}\right), \quad \text { as } m \rightarrow \infty,
\end{aligned}
$$

after approximating the $\nu_{q j}$ by replacing $g_{j}$ by $\lambda_{j}$, if necessary, and approximating sums by integrals.

Next, in view of the proofs for $A_{1}$ and $V\left(A_{2}\right)$ it is readily seen that

$$
V\left(A_{3}\right)=o\left(\frac{1}{m}\right) .
$$

We finally consider the probability orders of $A_{4}$ and $A_{5}$. We have

$$
A_{4}=O_{p}\left(\left(\frac{\log m}{m}\right)^{2 / 3}+\frac{1}{m^{1 / 2} n^{1 / 4}}\right)=o_{p}\left(\frac{1}{m^{1 / 2}}\right) .
$$


In case $\alpha=1$ in (2.6), this follows directly from the proof of (4.8) of Robinson (1995b). The same method of proof is also instrumental when $\psi(z)$ is nonlinear, but in addition one has to consider a Taylor approximation to $A_{4}$; the "linear" term will dominate, but the $I_{j}$ and $I_{\varepsilon j}$ also appear in the denominator, and here the Gaussianity is useful, c.f. Hurvich, Deo and Brodsky (1998). Finally because

$$
\begin{aligned}
f_{j}-f_{l} & =g_{j}^{1-2 H}\left\{h\left(\lambda_{j}\right)-h\left(\lambda_{l}\right)\right\}+h\left(\lambda_{l}\right)\left(g_{j}^{1-2 H}-g_{l}^{1-2 H}\right) \\
& =O\left(g_{j}^{1-2 H}\left(\frac{j}{n^{2}}+\frac{1}{j}\right)\right), \quad j-J+1<l<j,
\end{aligned}
$$

we have

$$
A_{5}=O_{p}\left(\frac{\log m}{m} \sum_{j}^{\prime}\left(\frac{j}{n^{2}}+\frac{1}{j}\right)\right)=O_{p}\left(\frac{m \log m}{n^{2}}+\frac{(\log m)^{2}}{m}\right) .
$$

\section{A Special Case}

It is of interest to specialize the formulae of Section 3 in case of the higherorder kernel (2.5) with $q \geq 2$, and the Box-Cox family (2.6), (2.7), focussing on the case (2.11) for $g$, so $G=0$.

Noting first that, for $c>-1$,

$$
\int_{0}^{1} u^{c} d u=\frac{1}{c+1}, \quad \int_{0}^{1} \log (u) u^{c} d u=-\left(\frac{1}{c+1}\right)^{2},
$$

we have under (2.5)

$$
\sum_{j=0}^{q} \frac{a_{j}}{2 j+1}=1
$$

from (2.1). Also

$$
\begin{aligned}
\int_{0}^{1} u^{2 i} k_{q}(u) d u & =\sum_{j=0}^{q} \frac{a_{j}}{(2 i+2 j+1)}, \\
\int_{0}^{1} \log (u) u^{2 i} k_{q}(u) d u & =-\sum_{j=0}^{q} \frac{a_{j}}{(2 i+2 j+1)^{2}},
\end{aligned}
$$


SO

$$
\begin{aligned}
U_{i q} & =-\sum_{j=0}^{q} a_{j}\left\{\frac{1}{(2 i+2 j+1)^{2}}-\frac{1}{2 i+2 j+1}\right\} \\
& =\sum_{j=0}^{q} a_{j} \frac{2 i+2 j}{(2 i+2 j+1)^{2}} .
\end{aligned}
$$

Thus, denoting by $D$ the $(q+1) \times(q+1)$ matrix with $(i, j)$ th element $2(i+j-2) /(2 i+2 j-3)^{2}$ for $i=1, \ldots, q, j=1, \ldots, q+1$ and $(q+1, j)$ th element $1 /(2 j-1), j=1, \ldots, q+1$, and by $d$ the $(q \times 1)$ th vector all of whose elements are zero except for the $(q+1)$ st, which is unity, and writing $a_{(q)}=\left(a_{0}, \ldots, a_{q}\right)^{\prime}$, we have

$$
a_{(q)}=-D^{-1} d .
$$

For example, we deduce that

$$
\begin{aligned}
& a_{(2)}=(0.45,9.91,-13.77)^{\prime}, \\
& a_{(3)}=(-0.15,34.69,-106.23,75.85)^{\prime}, \\
& a_{(4)}=(-0.57,64.71,-314.69,484.75,-236.79)^{\prime} .
\end{aligned}
$$

For any $q$ we have from (5.2)

$$
U_{q q}=U_{q q}^{*}=\sum_{j=0}^{q} a_{j} \frac{2 q+2 j}{(2 q+2 j+1)^{2}} .
$$

Further, because of (5.1) and also

$$
\int_{0}^{1}(\log u)^{2} u^{c} d u=\frac{2}{(c+1)^{3}}, \quad c>-1,
$$

we have

$$
\begin{aligned}
V_{q} & =V_{q}^{*}=\sum_{j=0}^{q} a_{j} \int_{0}^{1} u^{2 j}\left\{(\log u)^{2}+2 \log u+1\right\} d u \\
& =\sum_{j=0}^{q} a_{j}\left\{\frac{2}{(2 j+1)^{3}}-\frac{2}{(2 j+1)^{2}}+\frac{1}{2 j+1}\right\} \\
& =\sum_{j=0}^{q} a_{j} \frac{4 j^{2}+1}{(2 j+1)^{3}},
\end{aligned}
$$




$$
\begin{gathered}
W_{q}=W_{q}^{*}=\sum_{j=0}^{q} \sum_{l=0}^{q} a_{j} a_{l} \int_{0}^{1} u^{2 j+2 l}\left\{(\log u)^{2}+2 \log u+1\right\} d u \\
=\sum_{j=0}^{q} \sum_{l=0}^{q} a_{j} a_{l}\left\{\frac{2}{(2 j+2 l+1)^{3}}-\frac{2}{(2 j+2 l+1)^{2}}+\frac{1}{2 j+2 l+1}\right\} \\
=\sum_{j=0}^{q} \sum_{l=0}^{q} a_{j} a_{l} \frac{4(j+l)^{2}+1}{(2 j+2 l+1)^{3}}, \\
Y_{1 q}=\sum_{j=0}^{q} \frac{a_{j}}{2 j+5}-\left\{\sum_{j=0}^{q} \frac{a_{j}}{2 j+3}\right\}^{2} .
\end{gathered}
$$

Table A evaluates $U_{q q}, V_{q}, W_{q}$ and $Y_{1 q}$ for $q=1,2,3,4$.

Table A: $U_{q q}, V_{q}, W_{q}, Y_{1 q}$
\begin{tabular}{|l|l|l|l|l|}
\hline & $U_{q q}$ & $V_{q}$ & $W_{q}$ & $Y_{1 q}$ \\
\hline 1 & .2222 & 1 & 1 & .0889 \\
\hline $\mathrm{q}$ & -.0745 & .4125 & .9910 & -.0515 \\
\hline 3 & .0143 & .0089 & .8554 & -.0018 \\
\hline 4 & -.0005 & -.2066 & .5723 & .0011 \\
\hline
\end{tabular}

Next, specializing $\psi(z)$ to $\psi_{\alpha}(z)$ given by $(2.6),(2.7)$, note that $\psi_{\alpha}(z)$ has $u$ th derivative

$$
\begin{aligned}
\psi_{\alpha}^{(u)}(z) & =\frac{\Gamma(\alpha) z^{\alpha-u}}{\Gamma(\alpha-u+1)}, \quad u \geq 1, \quad 0<\alpha<1, \\
& =z^{-1}, \quad u=1, \quad \alpha=0, \\
& =(-1)^{u-1} \Gamma(u) z^{-u}, \quad u>1, \quad \alpha=0 \\
& =1, \quad u=1, \quad \alpha=1, \\
& =0, \quad u>1, \quad \alpha=1 .
\end{aligned}
$$

Thus because $E\left(Z_{J}^{\gamma}\right)=\Gamma(\gamma+J) /\left\{\Gamma(J) J^{\gamma}\right\}$ (see e.g. Johnson and Kotz 
(1970, p.168)) we deduce that

$$
\begin{aligned}
E\left(\left(C Z_{J}\right)^{u} \psi^{(u)}\left(C Z_{J}\right)\right) & =\frac{C^{\alpha} \Gamma(\alpha) \Gamma(\alpha+J)}{\Gamma(\alpha-u+1) \Gamma(J) J^{\alpha}}, \quad u \geq 1,0<\alpha<1, \\
& =(-1)^{u+1} u !, \quad u \geq 1, \alpha=0, \\
& =C, \quad u=1, \alpha=1, \\
& =0, \quad u>1, \quad \alpha=1 .
\end{aligned}
$$

Thus

$$
\begin{aligned}
R_{J}\left(\psi_{\alpha}\right) & =\frac{2 C^{\alpha} \Gamma(\alpha+J)}{\Gamma(J) J^{\alpha}}, \quad, 0<\alpha<1, \\
& =2, \alpha=0 \\
& =2 C, \quad \alpha=1 .
\end{aligned}
$$

\begin{tabular}{|c|c|c|c|c|}
\hline & .25 & .5 & .75 & 1 \\
\hline & .9064 & .8862 & .9191 & 1 \\
\hline & .9527 & .9400 & .9563 & 1 \\
\hline & .9685 & .9594 & .9702 & 1 \\
\hline & .9764 & .9693 & .9774 & 1 \\
\hline
\end{tabular}

Table B evaluates $R_{J}\left(\psi_{\alpha}\right) /\left(2 C^{\alpha}\right)=\Gamma(\alpha+J) / \Gamma(J) J^{\alpha}$ for $\alpha=0, .25, .5, .75,1$ and $J=1,2,3,4$.

Table B: $R_{J}\left(\psi_{\alpha}\right) /\left(2 C^{\alpha}\right)$

From Johnson and Kotz (1970, pp. 168, 181) we deduce that

$$
\begin{aligned}
Q_{J}(\psi) & =\frac{C^{2 \alpha}\left\{\Gamma(2 \alpha+J) / \Gamma(J)-\Gamma(\alpha+J)^{2} / \Gamma(J)^{2}\right\}}{\alpha^{2} J^{2 \alpha-1}}, 0<\alpha<1, \\
& =\left.J \frac{d^{2}}{d z^{2}} \log \Gamma(z)\right|_{z=J}, \quad \alpha=0, \\
& =C^{2}, \quad \alpha=1 .
\end{aligned}
$$

Table C evaluates $Q_{J}\left(\psi_{\alpha}\right) / C^{2}$ for $\alpha=0, .25, .5, .75,1$ and $J=1,2,3,4$. 
Table C: $Q_{J}\left(\psi_{\alpha}\right) / C^{2}$

$\alpha$

\begin{tabular}{|l|l|l|l|l|l|}
\hline & 0 & .25 & .5 & .75 & 1 \\
\hline 1 & 1.6459 & 1.0346 & .8584 & .8616 & 1 \\
\hline 2 & 1.2918 & 1.0328 & .9314 & .9259 & 1 \\
\hline 3 & 1.1873 & 1.0253 & .9553 & .9496 & 1 \\
\hline 4 & 1.1392 & 1.0202 & .9667 & .9618 & 1 \\
\hline
\end{tabular}

Finally we have

$$
\begin{aligned}
P_{i J}\left(\psi_{\alpha}\right) & =\frac{(2 \pi)^{2 i} C^{\alpha} \Gamma(\alpha) \Gamma(\alpha+J)}{\Gamma(J) J^{\alpha}} \sum_{u=1}^{i} \frac{1}{\Gamma(\alpha-u+1) u !} \sum_{r_{1}+\ldots+r_{u}=i}\left(\prod_{l=1}^{u} \frac{h_{r_{l}}}{\left(2 r_{l}\right) !}\right), 0<\alpha<1, \\
P_{i J}\left(\psi_{0}\right) & =(2 \pi)^{2 i} \sum_{u=1}^{i}(-1)^{u+1} \sum_{r_{1}+\ldots+r_{u}=i}\left(\prod_{l=1}^{u} \frac{h_{r_{l}}}{\left(2 r_{l}\right) !}\right) \\
P_{i J}\left(\psi_{1}\right) & =(2 \pi)^{2 i} \frac{h_{i}}{(2 i) !}
\end{aligned}
$$

Note that, for the Box-Cox class (2.6), (2.7), the formulae (3.9)-(3.17) are all invariant to $C$.

It is apparent that pooling does not affect the bias when $\alpha=0,1$, and out of these two cases it affects variance only when $\alpha=0$. We give asymptotic bias, variance and optimal $m$ for $\alpha=0,1$ with $J=1$ but allowing for a general $q$ th-order kernel and taking $G=0$, i.e. with $g$ given by (2.11). For $\alpha=0$ (log -periodogram estimation):

$$
\begin{gathered}
\text { Asybias }=(2 \pi)^{2 q} \frac{U_{q q}}{2 V_{q}} \sum_{u=1}^{q}(-1)^{u} \sum_{r_{i}+\ldots+r_{u}=q}\left(\prod_{l=1}^{u} \frac{h_{r_{l}}}{\left(2 r_{l}\right) !}\right)\left(\frac{m}{n}\right)^{2 q}, \\
\text { Asyvar }=\frac{\pi^{2}}{24} \cdot \frac{W_{q}}{V_{q}^{2}} \cdot \frac{1}{m}, \\
\widehat{m}=\left[\pi^{2} W_{q} /\left(24 q(2 \pi)^{4 q} U_{q q}^{2}\left\{\sum_{u=1}^{q}(-1)^{u} \sum_{r_{1}+\ldots+r_{u}=q}\left(\prod_{l=1}^{u} \frac{h_{r_{l}}}{\left(2 r_{l}\right) !}\right)\right\}^{2}\right)\right]^{1 /(4 q+1)} n^{4 q /(4 q+1)},
\end{gathered}
$$


while for $\alpha=1$ (local Whittle estimation):

$$
\begin{aligned}
\text { Asybias } & =-(2 \pi)^{2 q} \frac{U_{q q} h_{q}}{2(2 q) ! V_{q}}\left(\frac{m}{n}\right)^{2 q} \\
\text { Asyvar } & =\frac{W_{q}}{4 V_{q}^{2}} \frac{1}{m} \\
\widehat{m} & =\left[W_{q}\{(2 q) !\}^{2} /\left\{4 q(2 \pi)^{4 q} U_{q q}^{2} h_{q}^{2}\right\}\right]^{1 /(4 q+1)} n^{4 q /(4 q+1)} .
\end{aligned}
$$

\section{$6 \quad$ Finite Sample Properties}

To examine further the implications for both choice of kernel order, and choice of $\alpha$ within the Box-Cox class of M-estimates, a Monte Carlo study was carried out. Three types of Gaussian model were employed:

1. $\operatorname{FARIMA}(0, H-0.5,0): f(\lambda) \propto|\sin (\lambda / 2)|^{1-2 H} ;$

2. FARIMA $(1, H-0.5,0)$ with autoregressive (AR) coefficient 0.5: $f(\lambda) \propto$ $|\sin (\lambda / 2)|^{1-2 H}(1.25-\cos \lambda)^{-1}$

3. Gegenbauer FARIMA with poles at frequencies 0 and $\pi / 4$, of intensities $H / 2-0.25$ and 0.25 respectively: $f(\lambda) \propto|\sin (\lambda / 2)|^{1-2 H}$ $\times\{\sin (\lambda / 2+\pi / 8) \sin (\lambda / 2-\pi / 8)\}^{-0.5}$.

Model 1 is the simplest possible setting for comparisons across $H, \alpha$ and kernel order. In Model 2, the competing influence of an AR spectral peak near frequency zero is examined. The cyclic pole in Model 3 does not affect the asymptotic properties described in the previous section, but is liable to impact on finite sample behaviour to some degree (indeed disastrously if $m$ is chosen large enough). For each of these models three values of $H$ were used:

$$
H=0.25, \quad H=0.5, \quad H=0.75,
$$

corresponding respectively to moderate antipersistence, short memory, and moderate long memory. In each of the $3 \times 3=9$ cases, 1000 replications of series of length $n=1000$ were generated, according to the approach used in Robinson and Henry (1999) for Models 1 and 2, and Gray, Zhang and Woodward (1989) for Model 3. 
For each series generated, $4 \times 3 \times 2=24$ estimates $\widehat{H}_{q m}(\psi, g, k, 1)$ were computed, such that

$$
\begin{aligned}
& \psi(z)=\psi_{\alpha}(z), \quad \alpha=0,0.5,1 \\
& k(u)=(2.5), \quad q=1,2,3,4 ; \quad(\text { see }(2.18),(5.4)-(5.6)) ; \\
& g(\lambda)=(2.11), \quad(2.12) .
\end{aligned}
$$

Thus as well as versions of the familiar log periodogram and local Whittle estimates $(\alpha=0,1)$, we considered an intermediate, "square-root" estimate $(\alpha=0.5)$. The coefficients for the four kernels are given in (2.18), (5.4)(5.6); one expects, at least for large enough $n$, most scope for bias reduction when $g(\lambda)=(2.12)$ in case of Model 1, but not necessarily in Model 2. The bandwidth $m$ is determined separately in each case, according to an automatic, data-dependent, optimal procedure in which the $h_{i}$ are approximated by periodogram regression near zero frequency, extending the method proposed in Henry and Robinson (1996): their procedure covers the case $q=1$ only, regressing on a constant and $\lambda_{j}^{2}$, while for $q \geq 2$ we regress also on $\lambda_{j}^{2 k}, k=2, \ldots, q$. The constraint $m \leq 499$ was imposed since we introduced $m$ to satisfy $1<m<n / 2$.

Tables 1, 2 and 3 correspond to Models 1, 2 and 3 respectively, each Table being split into two sub-tables according to the choice of $g$. Within each subtable, for each combination of $H, \alpha$ and $q$, we give: (Monte Carlo) bias; two root mean squared errors (rmses), namely the Monte Carlo rmse followed by the theoretical asymptotic minimal rmse; two $m$ 's, namely the automatic $m$ averaged across Monte Carlo replications followed by the theoretical asymptotic optimal $m$. The theoretical asymptotic optimal $m$ and theoretical asymptotic minimal rmse were computed using formulae (3.12)-(3.14) and (3.15)-(3.17), respectively, and the numerical results of Section 5, with the $h_{i}$ being determined by expanding the appropriate $h(\lambda)$; these calculations were quite complicated.

The biases do not display very systematic behaviour, indeed since approximately optimal bandwidths were used, any improvements should appear in the Monte Carlo rmses only, and not in the biases. However we discuss first the Monte Carlo $m$. These increase monotonically in kernel order $q$. The averaged $m$ are smallest for $H=0.75$ (long memory); in the FARIMA $(1, H-0.5,0)$, they are larger for $H=0.5$ than for $H=0.25$, but the converse is true for the other models. There is little apparent sensitivity to $\alpha$ except in Model 2 (where an autoregressive component is included) and 
$m$ decreases in $\alpha$ in 17 out of 24 cases for kernel orders $q=1,2$, but decreases in $\alpha$ in 17 out of 24 cases for $q=3,4$. There is surprisingly little sensitivity of $m$ to $g$ even though, for FARIMA $(0, H-0.5,0)$, the $h_{i}$ are all zero under (2.12) and all non-zero under (2.11).

We now consider the Monte Carlo rmses. For Model 1, rmse is always minimized by kernel order $q=2$, while on the other hand $q=3$ performs worst in 16 out of 18 cases. For the $\operatorname{FARIMA}(1, H-0.5,0), q=1$ is always best when $H=0.25$ and $H=0.5$, and $q=4$ is worst in 15 out of 18 cases. On the other hand, $q=2$ is always most efficient when $H=0.75$, and it is especially notable that $q=1$ comes third in 2 cases and fourth in 2 cases, so that here the higher-order kernels with $q=3$ and 4 also afford some improvement over the unweighted estimate. This support for higher-order kernels is underlined by the fact that the long memory case is often the most interesting in practice. Finally, for the Gegenbauer Model 3, all higher-order kernel procedures are very inefficient, presumably because of a failure of the automatic bandwidth selection procedure to respond to leakage from the pole at frequency $\pi / 4$.

The rmse is noticeably sensitive to $\alpha$, increasing with $\alpha$ in 92 out of 144 cases. However, interestingly, in a number of cases the new, intermediate estimate with $\alpha=0.5$ performs best. Finally, there is no significant pattern of dependence of rmse's on $H$.

With respect to theoretical rmse and $m$, there are a few cases in which they are close to their Monte Carlo counterparts, but mostly discrepancies are considerable. Indeed, in Table 1 with $g(\lambda)=\lambda$ for $H=0.5$, and $g(\lambda)=2 \sin (\lambda / 2)$ for all $H$, we report rmse $=0, m=\infty$ (so we did not impose the constraint $m \leq 499$, employed in the Monte Carlo); all $h_{i}$ are zero, so the bias contribution to rmse is non-existent and rmse is minimized by $m=\infty$. These cases are degenerate, $x_{t}$ being white noise for $H=0.5$, while for $g(\lambda)=2 \sin (\lambda / 2)$ the semiparametric 'approximation' is actually the true model, at all frequencies. The theoretical rmses for $q=3$ help to explain the Monte Carlo ones, indeed they are much worse. This is largely due to the numerically tiny $V_{3}$ (see Table A); there is no reason why the factor $W_{q} / V_{q}^{2}$ in the asymptotic variance should be monotonic in $q$ here, due to the $(\log u+1)^{2}$ factor in the integrand of both $V_{q}$ and $W_{q}$, absent in case of higherorder kernels in nonparametric spectral and probability density estimation. 
Note that our results also reflect our particular, polynomial, construction of $k_{q}(u)$. The fact that the theoretical rmses increase over $q=1,2,4$ is also surprising, and is essentially due to the increase in $W_{q} / V_{q}^{2}$ overwhelming the decrease in $n^{-4 q /(4 q+1)}$ for our value of $n$; for sufficiently large $n$ this phenomenon would be reversed. The increase in theoretical optimal $m$ with $q$ is expected, but in Table 1 with $g(\lambda)=\lambda$ we have $m>n$ for $q \geq 2$ or $q \geq 3$. This is due in part to the small theoretical bias here, but also reflects the fact that our $n$ is not large enough for the asymptotic formulae in (3.12)-(3.14) to be very reliable.

To analyze Monte Carlo bias, a different experiment was run with fixed bandwidths $m=64,128$ and 256, and focusing only on the $\operatorname{FARIMA}(0,0.25,0)$ case. The Monte Carlo biases and rmses (based still on 1000 replications) are reported in Table 4 . In all cases, the best-performing bandwidth is undoubtedly $m=256$, which shows that the automatic procedure for $q=1$ tends to undersmooth.

As expected, the choices $q=1$ and $q=2$ are indistinguishable from the point of view of Monte Carlo rmse's when the bandwidth is the same. More surprisingly, Monte Carlo biases are also very similar. The sensitivity of bias to $\alpha$ is quite marked, on the other hand, increasing with $\alpha$ in 46 out of 48 cases, and this from slightly negative values when $\alpha=0$ to slightly positive values when $\alpha=1$ in 42 out of 48 cases. The rmse's decrease with $\alpha$ in 40 out of 48 cases, while of the 8 remaining cases, the increase is $2 \%$ or less.

Overall in this study, it is encouraging that the methods work better than the theoretical asymptotic minimal rmse and optimal $m$ might suggest. We find evidence that a kernel of order $q=2$ can improve estimation, but little that a larger order can exploit the local smoothness available in the models considered, at least for the sample size considered. The new estimate with $\alpha=0.5$ emerges as a useful choice, intermediate between the log periodogram and local Whittle estimates, and sometimes having better finite sample properties than either.

\section{Application to Intra-Day Foreign Exchange Volatility}

The focus of this application is the estimation of long memory in nonlinear transformations of intra-day Foreign Exchange (FX) rates. We study three 
sets of FX returns, on the DEM/USD (Deutschmark/Dollar), JPY/USD (Yen/Dollar) and GBP/DEM (Sterling/Dollar), covering the period from the beginning of October 1992 to the beginning of March 1993 (26 weeks, week-ends excluded) and first investigated in the context of long memory estimation by Henry and Payne (1997). These return series are filtered transcriptions of the tick-by-tick quotation series appearing on the Reuters FXFX page. Each quote encompasses a timestamp, bid and ask quotation pair, plus identifiers which allow one to determine the inputting bank and its location. In this study we ignore these identifications using the tick-by-tick data solely to construct a homogenous time-series in calendar time. The basic horizon over which we calculate returns is 10 minutes. This yields, for each currency, a time-series with $n=18720$ observations (130 days with 144 observations each).

The choice of these series was motivated by the extensively documented daily seasonality of absolute, squared and log squared returns, and other transformations. For an extensive description of this seasonal pattern and its market microstructural interpretations, see Payne (1996). This seasonal behaviour was modelled, albeit for different exchange rates, by a Gegenbauer model with possibly asymmetric poles at the daily frequency and its multiples, in Arteche (2000). It is therefore a series for which long memory analysis may be best performed by means of purely local methods, as opposed to those in Hurvich and Brodsky (2001) and Moulines and Soulier (1999), which appeal more to global smoothness. For all three series, the strongest seasonal feature is a large peak in the estimated spectrum at the daily frequency (in this case $2 \pi / 144$ ), corresponding to periodogram harmonic 130 , and at multiples of the daily frequency.

We estimated $H$ from $x_{t}=$ absolute returns, squared returns, and squarerooted absolute returns, using the same $\alpha, k$ and $g$, and the same type of automatic $m$, as in the previous section. Of course such $x_{t}$ are certainly not Gaussian, though theoretical evidence from, for example, Robinson (1995b), Robinson and Henry (1999) and Velasco (2000) suggests that this may not affect asymptotic properties. More seriously, Deo and Hurvich (2001) have pointed out the inconsistency between stochastic volatility models and differentiability of $h(\lambda)$ in (3.1), where $f(\lambda)$ is the spectral density of squares or other instantaneous nonlinear functions of the data. Arguing rather informally, in case we observe $y_{t}=\varepsilon_{t} \sigma_{t}$ where $\left\{\varepsilon_{t}\right\}$ is a sequence of zero-mean independent variates, independent also of the stationary sequence $\left\{\sigma_{t}\right\}$, then the $y_{t}$ are martingale differences and thus serially uncorrelated, and the spec- 
tral density of $x_{t}=\left|y_{t}\right|^{\beta}, \beta>0$, is of form

$$
f(\lambda)=\widetilde{f}(\lambda)+\frac{\tau^{2}}{2 \pi}
$$

where $\tilde{f}(\lambda)$ is the spectral density of $E\left|\varepsilon_{t}\right|^{\beta} E\left|\sigma_{t}\right|^{\beta}$ and $\tau^{2}=V\left(\left|\varepsilon_{t}\right|^{\beta}\right) E\left|\sigma_{t}\right|^{2 \beta}$. Then if $\widetilde{f}(\lambda)=C \lambda^{1-2 H} \widetilde{h}(\lambda)$, then no matter how smooth $\widetilde{h}(\lambda)$ is, $f(\lambda) \lambda^{2 H-1}$ satisfies a Lipschitz condition of order no greater than $2 H-1<1$. The same outcome occurs in more general 'signal-plus-noise' models. In this context there is no benefit to using higher-order kernels (though the issues of choice of $M$-estimate and pooling are still relevant). However, serial uncorrelatedness of $y_{t}=\varepsilon_{t} \sigma_{t}$ still holds if the independence properties of the $\varepsilon_{t}$ are relaxed such that $\mathrm{E}\left(\varepsilon_{t} \mid \varepsilon_{t-1}, \ldots, \sigma_{t}, \sigma_{t-1}, \ldots\right)=0$, almost surely, indeed the $y_{t}$ are still martingale differences, but this is insufficient for the representation (7.1), so the argument against the possibility of a smooth $h(\lambda)$ in (3.1) is no longer conclusive. More generally, though on the one hand many functions of mixing processes are mixing at the same rate, and on the other, taking a nonlinear instantaneous function of a Gaussian long memory process cannot increase memory, there seems to be no reason why there cannot exist $y_{t}$ that has short memory autocorrelation, and is even a martingale difference sequence, while nonlinear functions $x_{t}=r\left(y_{t}\right)$ not only have long memory but have spectral density of form (3.1) with $h(\lambda)$ satisfying (3.2) with $q \geq 1$. For example, if $x_{t}=y_{t}^{2}$ where $y_{t}$ is serially uncorrelated, $x_{t}$ has lag- $j$ autocorrelation $2\left(E y_{t}^{2}\right)^{2}+\operatorname{cum}\left(y_{t}, y_{t}, y_{t}, y_{t}\right)$ for $j=0$, and $\operatorname{cum}\left(y_{t}, y_{t}, y_{t+j}, y_{t+j}\right)$, for $j \neq 0$, where considerable generality is possible in the fourth cumulant function. In fact, even in (7.1) it is strictly speaking possible for $\widetilde{f}(\lambda)$ to include an additive component $-\tau^{2} /(2 \pi)$, such that $f(\lambda)$ has sufficient smoothness to justify use of higher-order kernels.

As results for the three transformed data series are similar, we include only those for absolute returns, in Table 5. We notice first that varying $g$ has no effect, and that the great similarities in results for the different exchange rates lend plausibility to the hypothesis of a common factor, which may be interpreted as resulting from a common information arrival process affecting volatility. All estimated values of $H$ fall within the interval $(0.5,1)$, and except when $\alpha=0$ and $q=3$, approximate $95 \%$ confidence intervals exclude $H=1 / 2$. Thus in genrral it appears that such a common factor might be well modelled by a stationary long memory component.

Looking at the results of Table 5 in more detail, we see that for $(\alpha, q) \neq$ $(0,3)$ all estimates of $H$ lie in the interval $(0.73,0.93)$. There is a clear in- 
crease in automatic bandwidths with $q$ and $\alpha$, which results in an increase in $\alpha$ of estimated $H$, while use of a higher order kernel reduces the estimates in 25 out of 27 cases. Very similar results, not reported here, obtain with deseasonalized spectra (using a double window deseasonalization procedure described in Henry and Payne (1997)). This confirms both that the automatic bandwidth selection procedure is insensitive to low leakage peaks in the spectrum (a rather undesirable feature), and that $M$-estimation of long memory (with or without higher-order kernels) is robust to the type of peaks present in the spectra of intra-day foreign exchange absolute returns. 


\section{$9 \quad$ References}

Andrews, D.W.K., Guggenberger, P., 2000. A bias-reduced log-periodogram estimator for the long memory parameter. Preprint, Yale University.

Andrews, D.W.K., Sun, Y., 2001. Local polynomial Whittle estimation of long-range dependence. Preprint, Yale University.

Arteche, J., 2000. Gaussian semiparametric estimation in seasonal/cyclical long memory time series. Mimeo, Euskal Erriko Univertsitatea.

Cogburn, I., Davis, H.T., 1974. Periodic splines and spectral estimation. Annals of Statistics 2, 1108-1126.

Deo, R.S., Hurvich, C.M., 2001. On the log periodogram regression estimator of the memory parameter in long memory stochastic volatility models. Econometric Theory 17, 686-710.

Geweke, J., Porter-Hudak, S., 1983. The estimation and application of long memory time series models. Journal of Time Series Analysis 4, 221-238.

Graf, H.-P., 1983. Long Range Correlations and Estimation of the SelfSimilarity Parameter. Dissertation ETH, Zurich.

Gray, H.L., Zhang, N.I., Woodward, W.A., 1989. On generalized fractional processes. Journal of Time Series Analysis 10, 233-257.

Hannan, E. J., 1957. The variance of the mean of a stationary process. Journal of the Royal Statistical Society Series B 19, 282-285.

Henry, M., Payne, R., 1997. An investigation of long range dependence in intra-day foreign exchange volatility. Discussion Paper 264, Financial Markets Group, London School of Economics.

Henry, M., Robinson, P.M., 1996. Bandwidth choice in Gaussian semiparametric estimation of long range dependence. Conference on Applied Probability and Time Series Analysis, Volume II: Time Series Analysis, In Memory of E.J. Hannan. Ed. by P.M. Robinson and M. Rosenblatt, Springer Verlag, New York, pp.220-232. 
Hurvich, C. M., Brodsky, J., 2001. Broadband semiparametric estimation of the memory parameter of a long-memory time series using fractional exponential models. Journal of Time Series Analysis. 22, 221-2496.

Hurvich, C.M., Deo, R. S., Brodsky, J., 1998. The mean squared error of Geweke and Porter-Hudak's estimation of the memory parameter of a long-memory time series. Journal of Time Series Analysis. 19, 19-46.

Johnson, N.L., Kotz, S. , 1970. Continuous Univariate Distributions, Volume 1. Wiley, New York.

Künsch, H., 1987. Statistical aspects of self-similar processes. In Proceedings of the First World Congress of the Bernoulli Society, 67-74. VNU Science Press.

Lobato, I., 1999, A semiparametric two-step estimator in a multivariate long memory model. Journal of Econometrics 90, 129-153.

Lobato, I., Robinson, P.M., 1998. A nonparametric test for I(0). Review of Economic Studies 65, 475-495.

Moulines, E., Soulier, P., 1999. Broadband log periodogram regression of time series with long range dependence. Annals of Statistics 27, 14151439 .

Parzen, E., 1957. On consistent estimates of the spectrum of a stationary time series. Annals of Mathematical Statistics 28, 329-348.

Payne, R., 1996. Announcement effects and seasonality in the intra-day foreign exchange market. Discussion Paper 238, Financial Markets Group, London School of Economics.

Phillips, P.C.B., 1987. Time series regression with a unit root. Econometrica 55, 277-301.

Phillips, P.C.B., 1991. Spectral regression for cointegrated time series. In W.A Barnett, J. Powell and G. Tauchen (eds.) Nonparametric and Semiparametric Methods in Econometrics and Statistics, Cambridge: Cambridge University Press.

Robinson, P.M., 1994. Semiparametric analysis of long-memory time series. Annals of Statistics 22, 515-539. 
Robinson, P.M., 1995a. Log-periodogram regression of time series with long range dependence. Annals of Statistics 23, 1048-1072.

Robinson, P.M., 1995b. Gaussian semiparametric estimation of long range dependence. Annals of Statistics 23, 1630-1661.

Robinson, P.M., Henry M., 1999. Long and short memory conditional heteroscedasticity in estimating the memory parameters of levels. Econometric Theory 15, 299-336.

Velasco, C., 1999. Non-stationary log-periodogram regression. Journal of Econometrics 91, 525-371.

Velasco, C., 2000. Non-Gaussian log-periodogram regression. Econometric Theory 16, 44-79. 
Table 1: $\operatorname{FARIMA}(0, \mathrm{H}-1 / 2,0)$

For each combination of $H, \alpha$ and $q$ we report Monte Carlo bias; Monte Carlo rmse followed by theoretical asymptotic minimal rmse; Monte Carlo automatic $m$ followed by theoretical asymptotic optimal $m$. $g(\lambda)=\lambda:$

\begin{tabular}{|c|c|c|c|c|c|c|c|c|c|c|}
\hline \multirow[t]{3}{*}{$\alpha$} & \multirow[t]{3}{*}{ q } & \multicolumn{9}{|c|}{$\mathrm{H}$} \\
\hline & & \multicolumn{3}{|c|}{0.25 (antipersistence) } & \multicolumn{3}{|c|}{0.50 (short memory) } & \multicolumn{3}{|c|}{0.75 (long memory) } \\
\hline & & bias & rmse & $\mathrm{m}$ & bias & rmse & $\mathrm{m}$ & bias & rmse & $\mathrm{m}$ \\
\hline \multirow{4}{*}{0} & 1 & -.006 & .057 .035 & 217415 & -.006 & .0530 & $241 \infty$ & -.007 & .063 .035 & 184415 \\
\hline & 2 & .002 & .044 .054 & 293926 & -.002 & .0440 & $308 \infty$ & -.010 & .046 .054 & 273926 \\
\hline & 3 & .126 & .3501 .95 & 4031258 & .016 & .3040 & $425 \infty$ & -.070 & .2841 .95 & 3721258 \\
\hline & 4 & -.022 & .064 .058 & 4781760 & -.006 & .0620 & $493 \infty$ & .004 & .056 .058 & 4521760 \\
\hline \multirow{4}{*}{.5} & 1 & .001 & .048 .032 & 209376 & -.004 & .0440 & $225 \infty$ & -.004 & .055 .033 & 174376 \\
\hline & 2 & .004 & -0.038 .043 & 2801090 & -.001 & .0340 & $292 \infty$ & -.004 & .039.051 & 240787 \\
\hline & 3 & .116 & .2451 .66 & 4171356 & .019 & .1710 & $423 \infty$ & -.009 & .0991 .83 & 3731108 \\
\hline & 4 & -.015 & .050 .049 & 4861871 & .001 & .0500 & $496 \infty$ & .018 & .052 .053 & 4591585 \\
\hline \multirow{4}{*}{1} & 1 & .002 & .051.029 & 202376 & -.005 & .0440 & $227 \infty$ & -.001 & .055 .029 & 170376 \\
\hline & 2 & .005 & .038 .032 & 2761192 & -.001 & .0350 & $290 \infty$ & -.002 & .037.047 & 249732 \\
\hline & 3 & .165 & .2731 .39 & 4041514 & .086 & .1790 & $414 \infty$ & .027 & .0961 .68 & 3771031 \\
\hline & 4 & .291 & .458.041 & 4992099 & .048 & .1600 & $499 \infty$ & .047 & .089. & 4001486 \\
\hline
\end{tabular}

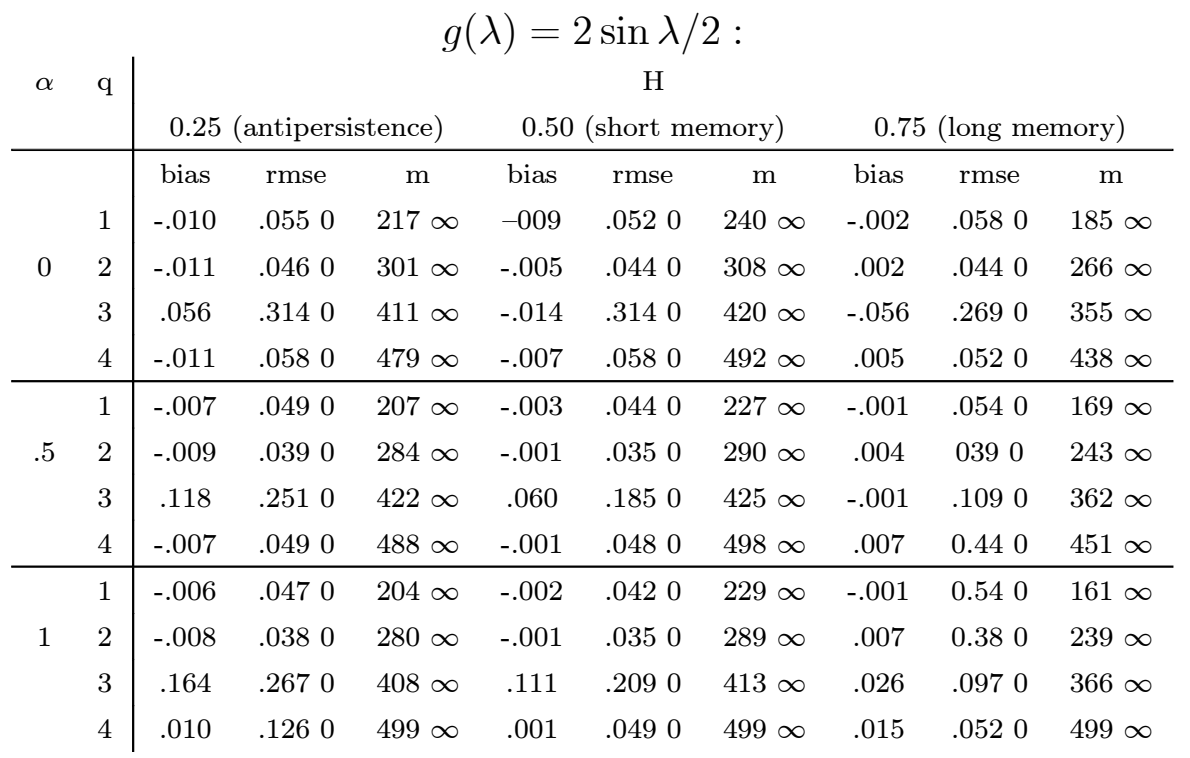


Table 2: $\operatorname{FARIMA}(1, H-1 / 2,0)$

For each combination of $H, \alpha$ and $q$ we report Monte Carlo bias; Monte Carlo rmse followed by theoretical asymptotic minimal rmse; Monte Carlo automatic $m$ followed by theoretical asymptotic optimal $m$. $g(\lambda)=\lambda$ :

\begin{tabular}{|c|c|c|c|c|c|c|c|c|c|c|}
\hline \multirow[t]{4}{*}{$\alpha$} & \multirow[t]{3}{*}{$\mathrm{q}$} & \multicolumn{9}{|c|}{$\mathrm{H}$} \\
\hline & & \multicolumn{3}{|c|}{0.25 (antipersistence) } & \multicolumn{3}{|c|}{0.50 (short memory) } & \multicolumn{3}{|c|}{0.75 (long memory) } \\
\hline & & bias & rmse & $\mathrm{m}$ & bias & rmse & $\mathrm{m}$ & bias & rmse & $\mathrm{m}$ \\
\hline & 1 & .126 & .152 .088 & $170 \quad 67$ & .040 & .098. & $83 \quad 67$ & -.010 & .135 .087 & $48 \quad 67$ \\
\hline \multirow[t]{4}{*}{0} & 2 & .215 & .226 .154 & 231114 & .098 & .118 .154 & 132114 & .032 & 154 & 77114 \\
\hline & 3 & .138 & .2875 .70 & 306148 & .146 & .1675 .70 & 170148 & .009 & .1925 .70 & 96148 \\
\hline & 4 & .215 & .225 .170 & 406204 & .235 & . 170 & 230204 & .047 & .172 .170 & 127204 \\
\hline & 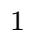 & .106 & .135 .081 & 14260 & .020 & .092. 081 & 6261 & -.040 & .176 .081 & 3461 \\
\hline \multirow[t]{4}{*}{.5} & 2 & .211 & .222 .145 & 21799 & .090 & .112 .145 & 12299 & .033 & 145 104 & $70 \quad 99$ \\
\hline & 3 & .084 & .1535 .35 & 309130 & .162 & .1685 .35 & 168130 & .006 & .1605 .35 & 94130 \\
\hline & 4 & .232 & .237 .158 & 423181 & .246 & .261 .158 & 229181 & .032 & .154 .158 & 126181 \\
\hline & 1 & .087 & .118 .072 & 12060 & .006 & .103 .072 & $\begin{array}{ll}51 & 61\end{array}$ & -.057 & .208 .072 & $25 \quad 61$ \\
\hline \multirow[t]{3}{*}{1} & 2 & .220 & 133 229 & 21493 & .107 & 133. 123 & 13293 & .059 & 132 103 & 8593 \\
\hline & 3 & .104 & .1764 .92 & 360121 & .163 & .1714 .91 & 255121 & .180 & .1854 .91 & 184121 \\
\hline & 4 & .230 & .232 .145 & 499169 & .245 & 145. 249 & 478169 & .235 & .235. 145 & 378169 \\
\hline
\end{tabular}

$g(\lambda)=2 \sin \lambda / 2:$

\begin{tabular}{|c|c|c|c|c|c|c|c|c|c|c|}
\hline \multirow[t]{2}{*}{$\alpha$} & \multirow[t]{2}{*}{$\mathrm{q}$} & \multicolumn{9}{|c|}{$\mathrm{H}$} \\
\hline & & \multicolumn{3}{|c|}{0.25 (antipersistence) } & \multicolumn{3}{|c|}{0.50 (short memory) } & \multicolumn{3}{|c|}{0.75 (long memory) } \\
\hline \multirow{5}{*}{0} & & bias & rmse & $\mathrm{m}$ & bias & rmse & $\mathrm{m}$ & bias & rmse & $\mathrm{m}$ \\
\hline & 1 & .129 & .157 .088 & 17467 & .035 & .096 .088 & $82 \quad 67$ & -.021 & .144 .088 & $47 \quad 67$ \\
\hline & 2 & .218 & .231 .137 & 233113 & .099 & .120 .137 & 131113 & .027 & .113 .137 & 75113 \\
\hline & 3 & .030 & .1761 .78 & 3091239 & .141 & .1711 .78 & 1691239 & .001 & .1871 .78 & 941239 \\
\hline & 4 & .213 & .225 .086 & $408 \quad 657$ & .234 & .251 .086 & 228657 & .033 & .176 .086 & 125657 \\
\hline \multirow{4}{*}{.5} & 1 & .100 & .128 .081 & $136 \quad 61$ & .014 & .096 .081 & 6061 & -.044 & .170 .081 & 3361 \\
\hline & 2 & .208 & .221 .129 & 21399 & .085 & .107 .129 & 11999 & .033 & .102 .129 & 6899 \\
\hline & 3 & .086 & .1391 .61 & 3041173 & .161 & .1751 .61 & 1641173 & .009 & .1501 .61 & 911173 \\
\hline & 4 & .236 & .242 .077 & $416 \quad 622$ & .247 & .267 .077 & $223 \quad 622$ & .031 & . 158.077 & 122622 \\
\hline \multirow{4}{*}{1} & 1 & .085 & .116 .072 & 11961 & .001 & .115.072 & $48 \quad 61$ & -.059 & .211 .072 & $25 \quad 61$ \\
\hline & 2 & .218 & .227 .118 & 21293 & .100 & 118. 119 & 12893 & .060 & .118 & 8493 \\
\hline & 3 & .075 & .1041 .42 & 3581172 & .164 & .1731 .42 & 2491172 & .184 & .1891 .42 & 1831172 \\
\hline & 4 & .228 & .232.069 & $472 \quad 622$ & .245 & .250 .069 & 472622 & .236 & 237.069. & 377622 \\
\hline
\end{tabular}


Table 3: Gegenbauer

For each combination of $H, \alpha$ and $q$ we report Monte Carlo bias; Monte Carlo rmse followed by theoretical asymptotic minimal rmse; Monte Carlo automatic $m$ followed by theoretical asymptotic optimal $m$. $g(\lambda)=\lambda:$

\begin{tabular}{|c|c|c|c|c|c|c|c|c|c|c|}
\hline \multirow[t]{4}{*}{$\alpha$} & \multirow[t]{3}{*}{ q } & \multicolumn{9}{|c|}{$\mathrm{H}$} \\
\hline & & \multicolumn{3}{|c|}{0.25 (antipersistence) } & \multicolumn{3}{|c|}{0.50 (short memory) } & \multicolumn{3}{|c|}{0.75 (long memory) } \\
\hline & & bias & rmse & $\mathrm{m}$ & bias & rmse & $\mathrm{m}$ & bias & rmse & $\mathrm{m}$ \\
\hline & 1 & 0.009 & .057 .074 & 21595 & -.009 & .052 .074 & 23094 & -.008 & .060 .074 & 19193 \\
\hline \multirow[t]{4}{*}{0} & 2 & -.001 & .044 .135 & 296148 & -.002 & .043 .135 & 305148 & -.007 & .046 .135 & 269148 \\
\hline & 3 & -.130 & .3635 .10 & 411185 & -.022 & .2965 .10 & 421185 & -.068 & .2765 .10 & 368185 \\
\hline & 4 & -.025 & .066 .153 & 482249 & -.005 & .062 .113 & 490249 & .009 & .057 .153 & 451249 \\
\hline & 1 & -.003 & .050 .068 & 20486 & -.005 & .044 .068 & $228 \quad 85$ & -.001 & .052 .069 & $178 \quad 84$ \\
\hline \multirow[t]{4}{*}{.5} & 2 & -.001 & .038 .125 & 279133 & -.003 & .034 .125 & 296133 & -.002 & .037 .125 & 252133 \\
\hline & 3 & .123 & .2474 .70 & 416169 & .026 & .1794 .70 & 427168 & -.001 & .0984 .70 & 379168 \\
\hline & 4 & -.019 & .051 .140 & 485230 & -.003 & .051 .141 & 498230 & .022 & .055 .141 & 465229 \\
\hline & 1 & .001 & .046 .060 & 20086 & -.004 & .044 .061 & $223 \quad 85$ & -.001 & .052 .161 & 16384 \\
\hline \multirow[t]{3}{*}{1} & 2 & .002 & .036. & 276128 & -.001 & .034. 113 & 288127 & -.002 & .037 .114 & 243127 \\
\hline & 3 & .171 & .2754 .26 & 405161 & .082 & .1794 .26 & 413161 & .034 & .0964 .27 & 372161 \\
\hline & 4 & .254 & .431 .127 & 499220 & .053 & .165 .127 & 499220 & .041 & .085 .127 & 499219 \\
\hline
\end{tabular}

$g(\lambda)=2 \sin \lambda / 2:$

\begin{tabular}{|c|c|c|c|c|c|c|c|c|c|c|}
\hline \multirow[t]{4}{*}{$\alpha$} & \multirow[t]{3}{*}{ q } & \multicolumn{9}{|c|}{$\mathrm{H}$} \\
\hline & & \multicolumn{3}{|c|}{0.25 (antipersistence) } & \multicolumn{3}{|c|}{0.50 (short memory) } & \multicolumn{3}{|c|}{0.75 (long memory) } \\
\hline & & bias & rmse & $\mathrm{m}$ & bias & rmse & $\mathrm{m}$ & bias & rmse & $\mathrm{m}$ \\
\hline & 1 & -.014 & $.057 \quad .074$ & 21994 & -.007 & .050 .074 & 24194 & -.006 & .063 .074 & 18194 \\
\hline \multirow[t]{4}{*}{0} & 2 & -.011 & .047 .118 & 302150 & -.004 & .043. 118 & 307150 & .001 & .047. 118 & 259150 \\
\hline & 3 & .033 & .2981 .62 & 4121498 & -.010 & .3151 .62 & 4211498 & -.070 & .2741 .62 & 3521498 \\
\hline & 4 & -.015 & .060 .078 & 480795 & -.005 & .057 .078 & 491795 & -.002 & .055 .078 & 436795 \\
\hline & 1 & -.005 & .047 .068 & 20185 & -.007 & .044 .068 & $230 \quad 85$ & -.003 & .052 .068 & 17185 \\
\hline \multirow[t]{4}{*}{.5} & 2 & -.008 & .037 .110 & 278134 & -.004 & .035 .110 & 296134 & .001 & .037 .110 & 246134 \\
\hline & 3 & .112 & .2481 .46 & 4121417 & .047 & .1871 .46 & 4261417 & -.013 & .1121 .46 & 3701417 \\
\hline & 4 & -.007 & .038 .071 & 485752 & -.003 & .047 .071 & 498752 & .005 & .043 .071 & 451752 \\
\hline & 1 & -.004 & .050 .061 & 20185 & -.002 & .044 .061 & 22185 & .001 & .059.061 & 16185 \\
\hline \multirow[t]{3}{*}{1} & 2 & -.001 & .039. 100 & 276128 & -.001 & .036. 100 & 289125 & .008 & 100. 040 & 240128 \\
\hline & 3 & .185 & .2921 .30 & 4041417 & .113 & .2081 .30 & 4121417 & .024 & .0981 .30 & 3671417 \\
\hline & 4 & .018 & .141 .063 & 499752 & .003 & .051.063 & 499752 & .013 & .052 .064 & 499752 \\
\hline
\end{tabular}


Table 4: FARIMA(0,H-1/2,0) and Fixed Bandwidths

For each combination of $H, \alpha$ and $q$ and $m$ we report Monte Carlo bias and Monte Carlo rmse.

$g(\lambda)=\lambda:$

\begin{tabular}{|c|c|c|c|c|c|c|c|}
\hline \multirow[t]{3}{*}{$\alpha$} & \multirow[t]{3}{*}{$q$} & \multicolumn{6}{|c|}{$m$} \\
\hline & & \multicolumn{2}{|c|}{64} & \multicolumn{2}{|c|}{128} & \multicolumn{2}{|c|}{256} \\
\hline & & bias & rmse & bias & rmse & bias & rmse \\
\hline \multirow{4}{*}{0} & 1 & -0.007 & 0.111 & -0.004 & 0.069 & -0.004 & 0.045 \\
\hline & 2 & -0.006 & 0.110 & -0.003 & 0.069 & -0.005 & 0.045 \\
\hline & 3 & -0.016 & 0.123 & -0.002 & 0.072 & -0.001 & 0.057 \\
\hline & 4 & -0.010 & 0.114 & -0.023 & 0.160 & 0.003 & 0.044 \\
\hline \multirow{4}{*}{0.5} & 1 & 0.001 & 0.080 & 0.006 & 0.051 & 0.001 & 0.035 \\
\hline & 2 & 0.002 & 0.080 & 0.006 & 0.051 & 0.001 & 0.035 \\
\hline & 3 & -0.006 & 0.085 & 0.010 & 0.057 & 0.008 & 0.048 \\
\hline & 4 & -0.001 & 0.081 & 0.013 & 0.131 & 0.002 & 0.035 \\
\hline \multirow{4}{*}{1} & 1 & 0.008 & 0.079 & 0.008 & 0.052 & 0.002 & 0.034 \\
\hline & 2 & 0.009 & 0.079 & 0.008 & 0.052 & 0.001 & 0.035 \\
\hline & 3 & -0.001 & 0.083 & 0.012 & 0.058 & 0.010 & 0.047 \\
\hline & 4 & 0.004 & 0.079 & 0.028 & 0.131 & 0.002 & 0.034 \\
\hline
\end{tabular}

$g(\lambda)=2 \sin \lambda / 2:$

\begin{tabular}{|c|c|c|c|c|c|c|c|}
\hline \multirow[t]{3}{*}{$\alpha$} & \multirow[t]{3}{*}{$q$} & \multicolumn{6}{|c|}{$m$} \\
\hline & & \multicolumn{2}{|c|}{64} & \multicolumn{2}{|c|}{128} & \multicolumn{2}{|c|}{256} \\
\hline & & bias & rmse & bias & rmse & bias & rmse \\
\hline \multirow{5}{*}{0} & 1 & -0.014 & 0.107 & -0.005 & 0.069 & 0.006 & 0.049 \\
\hline & 2 & -0.013 & 0.107 & -0.004 & 0.068 & 0.005 & 0.049 \\
\hline & 3 & 0.024 & 0.119 & 0.003 & 0.071 & 0.008 & 0.063 \\
\hline & 4 & -0.017 & 0.111 & -0.028 & 0.161 & 0.007 & 0.049 \\
\hline & 1 & 0.005 & 0.078 & 0.006 & 0.054 & 0.007 & 0.035 \\
\hline \multirow[t]{4}{*}{0.5} & 2 & 0.006 & 0.078 & 0.007 & 0.054 & 0.007 & 0.036 \\
\hline & 3 & -0.003 & 0.084 & 0.012 & 0.061 & 0.009 & 0.048 \\
\hline & 4 & 0.002 & 0.079 & 0.008 & 0.124 & 0.008 & 0.036 \\
\hline & 1 & 0.010 & 0.077 & 0.014 & 0.052 & 0.010 & 0.036 \\
\hline \multirow[t]{3}{*}{1} & 2 & 0.011 & 0.077 & 0.014 & 0.051 & 0.010 & 0.037 \\
\hline & 3 & 0.003 & 0.082 & 0.018 & 0.058 & 0.013 & 0.050 \\
\hline & 4 & 0.007 & 0.078 & 0.031 & 0.133 & 0.010 & 0.037 \\
\hline
\end{tabular}


Table 5: Foreign Exchange Absolute Returns

For each combination of $\alpha$ and $q$ we report the estimate $\widehat{H}$ and automatic $m$.

$g(\lambda)=\lambda:$

\begin{tabular}{|c|c|c|c|c|c|c|c|}
\hline \multirow[t]{2}{*}{$\alpha$} & \multirow[t]{2}{*}{$q$} & \multicolumn{2}{|c|}{ Deutsch Mark } & \multicolumn{2}{|c|}{ Sterling } & \multicolumn{2}{|c|}{ Yen } \\
\hline & & $\widehat{H}$ & $m$ & $\widehat{H}$ & $m$ & $\widehat{H}$ & $m$ \\
\hline \multirow{4}{*}{0} & 1 & 0.82 & 492 & 0.73 & 495 & 0.81 & 487 \\
\hline & 2 & 0.77 & 1228 & 0.73 & 1239 & 0.74 & 1210 \\
\hline & 3 & 0.56 & 1609 & 0.63 & 1623 & 0.53 & 1586 \\
\hline & 4 & 0.94 & 2135 & 0.81 & 2154 & 0.74 & 2105 \\
\hline \multirow{4}{*}{0.5} & 1 & 0.87 & 902 & 0.84 & 890 & 0.82 & 891 \\
\hline & 2 & 0.77 & 2796 & 0.76 & 2796 & 0.78 & 2812 \\
\hline & 3 & 0.75 & 2396 & 0.76 & 2422 & 0.77 & 2390 \\
\hline & 4 & 0.73 & 2887 & 0.72 & 2815 & 0.77 & 2826 \\
\hline \multirow{4}{*}{1} & 1 & 0.93 & 1361 & 0.93 & 1273 & 0.89 & 1379 \\
\hline & 2 & 0.88 & 2145 & 0.89 & 2065 & 0.86 & 2167 \\
\hline & 3 & 0.84 & 3208 & 0.85 & 3160 & 0.84 & 3299 \\
\hline & 4 & 0.81 & 7298 & 0.82 & 6480 & 0.83 & 6578 \\
\hline
\end{tabular}

$g(\lambda)=2 \sin \lambda / 2:$

\begin{tabular}{|c|c|c|c|c|c|c|c|}
\hline$\alpha$ & $q$ & Deut & Mark & Ste & ing & & \\
\hline & & $\widehat{H}$ & $m$ & $\widehat{H}$ & $m$ & $\widehat{H}$ & $m$ \\
\hline & 1 & 0.82 & 492 & 0.73 & 496 & 0.81 & 487 \\
\hline 0 & 2 & 0.77 & 1229 & 0.73 & 1241 & 0.74 & 1211 \\
\hline & 3 & 0.57 & 1611 & 0.63 & 1625 & 0.56 & 1588 \\
\hline & 4 & 0.94 & 2138 & 0.81 & 2156 & 0.74 & 2108 \\
\hline & 1 & 0.87 & 903 & 0.84 & 892 & 0.82 & 893 \\
\hline 0.5 & 2 & 0.77 & 2806 & 0.76 & 2806 & 0.79 & 2822 \\
\hline & 3 & 0.75 & 2400 & 0.76 & 2412 & 0.77 & 2384 \\
\hline & 4 & 0.73 & 2892 & 0.72 & 2834 & 0.78 & 2831 \\
\hline & 1 & 0.93 & 1364 & 0.93 & 1276 & 0.89 & 1382 \\
\hline 1 & 2 & 0.88 & 2159 & 0.89 & 2078 & 0.86 & 2181 \\
\hline & 3 & 0.85 & 3213 & 0.85 & 3165 & 0.84 & 3304 \\
\hline & 4 & 0.81 & 7308 & 0.82 & 6489 & 0.84 & 6587 \\
\hline
\end{tabular}

